\title{
Neue Perspektiven für die Aufklärung von genetischen Krankheiten
}

\author{
H.-H. Ropers \\ Max-Planck-Institut für molekulare Genetik, Berlin
}

Korrespondenz an: Prof. Dr. H.-Hilger Ropers, Max-Planck-Institut für molekulare Genetik, Ihnestraße 73, D-14195 Berlin; E-Mail: ropers@molgen.mpg.de

Eingegangen: 4. April 2008

Online First 24 April 2008

Schlüsselwörter: genetische Krankheiten, single nucleotide polymorphism, SNPs, genetische Risikofaktoren, multifaktorielle Krankheiten.

Zusammenfassung: Seit etwa 15 Jahren hat sich die Genomforschung auf die Suche nach genetischen Risikofaktoren für häufige Krankheiten konzentriert, jedoch ohne großen Erfolg. Erst seit kurzem scheint sich das Blatt zu wenden, vor allem durch die Einführung von hochdichten DNS-Rastern zur Typisierung von „single nucleotide polymorphisms“ (SNPs) und durch Untersuchung sehr viel größerer Kohorten als bisher. Die meisten der bisher gefundenen genetischen Risikofaktoren sind jedoch nur für einen kleinen Teil des gesamten genetischen Risikos verantwortlich und ihr diagnostischer Wert ist vernachlässigbar. Es gibt Grund zu der Annahme, dass die Komplexität vieler „multifaktorieller“ Krankheiten primär auf genetische Heterogenität zurückgeht, also auf Defekte in verschiedenen Genen, welche dieselbe Krankheit verursachen. Darüber hinaus konnten neu auftretende submikroskopische Deletionen und Duplikationen als eine wichtige, bisher unbekannte Ursache für geistige Behinderung und andere komplexe Krankheiten identifiziert werden, und in mehreren Fällen konnten die damit assoziierten klinischen Befunde auf Störungen einzelner Gene zurückgeführt werden. Diese Befunde sprechen dafür, die Untersuchung der bisher vernachlässigten monogenen Krankheiten in den Mittelpunkt der Genomforschung zu stellen. U. a. durch die Einführung neuartiger Hochdurchsatz-Sequenziertechnologien ist die Aufklärung von Erbkrankheiten viel einfacher und billiger geworden mit weit reichenden Konsequenzen für die Diagnostik und Prävention genetisch bedingter Störungen, aber auch für die Krankenversorgung allgemein (Ropers, 2007).

\section{Einführung}

Bis Anfang der 90iger Jahre wurde der Vorschlag, das gesamte menschliche Genom zu sequenzieren, mit der Erwartung begründet, dieses Projekt werde zur Aufklärung aller bekannten genetisch bedingten Krankheiten führen (Guyer und Collins,
1993). Optimistische Vorhersagen führender Genomforscher zur Bedeutung der Genomsequenzierung für die Aufklärung häufiger Krankheiten wie Herzinfarkt, Schlaganfall, Demenzkrankheiten, psychiatrische Störungen, Asthma und Krebs haben die Erwartungen der Öffentlichkeit danach weiter angeheizt. Für die Pharmaindustrie und für die Politik waren diese Prognosen außerordentlich attraktiv. Dies war der Grund dafür, warum die Suche nach den genetischen Ursachen komplexer Krankheiten in der Genomforschung weltweit Priorität bekommen hat.

\section{Genetische Risikofaktoren für komplexe Krankheiten: Licht am Ende des Tunnels?}

Unter der Annahme, dass die meisten häufigen Krankheiten multifaktoriell sind, d.h. dass sie auf ein Zusammenspiel genetischer und nicht-genetischer Faktoren zurückgehen und dass genetisch bedingte Risikofaktoren für häufige Krankheiten evolutionär alt sein müssen, sind im Rahmen von industrie- und regierungsfinanzierten Forschungsprogrammen Milliarden für die Suche nach DNS-Varianten im menschlichen Genom ausgegeben worden, die bei Patienten mit komplexen Krankheiten häufiger als bei Gesunden vorkommen. Derartige genomweite Assoziationsstudien haben jedoch häufig zu widersprüchlichen Befunden geführt. Dafür wurden in den meisten Fällen zu kleine Kohorten und eine zu geringe Dichte genetischer Marker verantwortlich gemacht.

Neue Studien haben gezeigt, dass schädliche Mutationen in der menschlichen Bevölkerung schnell eliminiert werden; deshalb ist es unwahrscheinlich, dass evolutionär alte Mutationen als häufige Polymorphismen in der menschlichen Bevölkerung überlebt haben (Kryukoe et al., 2007). Dies spricht dafür, daß die meisten genetischen Risikofaktoren für komplexe Krankheiten sehr selten sind, und diese Annahme wird durch neuere empirische Daten gestützt (Cohen et al., 2004).

Die Identifikation seltener Risiko-Allele, entweder direkt oder durch Assoziation, erfordert ein dichtes Netzwerk poly- 
morpher genetischer Marker. Eng benachbarte genetische Marker werden oft als evolutionär konservierte Haplotypen vererbt. Um die Auflösung von Assoziationsstudien zu maximieren und die Zahl der dafür erforderlichen Marker zu begrenzen, hat das internationale HapMap-Projekt (Zaitlen et al., 2007) etwa sechs Millionen verschiedene SNP-Marker identifiziert und das Kopplungsungleichgewicht zwischen diesen Markern charakterisiert. Die ursprüngliche Idee dieses Vorhabens bestand darin, den Aufwand für die genomweite SNP-Typisierung auf den Nachweis einer überschaubaren Zahl solcher Haplotyp-Blöcke zu reduzieren. Es hat sich jedoch herausgestellt, daß für die Erkennung dieser Haplotyp-Blöcke im ganzen Genom noch immer etwa 500000 SNP-Marker typisiert werden müssen, viel mehr als ursprünglich erwartet. Dennoch sind derartige Untersuchungen jetzt praktisch durchführbar, weil es seit kurzem DNA-Chips gibt, mit deren Hilfe mehr als 500000 SNPs in einem einzigen Experiment typisiert werden können. SNP-Typisierungen mithilfe derartiger DNA-Chips und die Analyse sehr großer Kohorten von Patienten und Kontrollen haben die Auflösung von genomweiten Assoziationsstudien entscheidend gesteigert. In jüngster Zeit hat dies zur Identifikation von genetischen Risikofaktoren für verschiedene komplexe Krankheiten geführt, einschließlich Typ II Diabetes, Herzinfarkt, Prostatakarzinom, Morbus Crohn und Fettleibigkeit (McPherson et al., 2007; Helgadottir et al., 2007; Rioux et al., 2007; Haiman et al., 2007; Gudmundsson et al., 2007; Yeager et al., 2007; Frayling et al., 2007; Dina et al., 2007). Darüber hinaus sind Pooling-Strategien entwickelt worden, welche die Kosten derartiger Untersuchungen drastisch gesenkt haben (Pearson et al., 2007).

\section{Die meisten bekannten Risikofaktoren sind für die Diagnostik ohne Bedeutung.}

Aufgrund der langen, ergebnislosen Suche nach solchen Risikofaktoren sind diese Ergebnisse von den beteiligten Forschern und der Öffentlichkeit mit Begeisterung und Erleichterung quittiert worden. Angesichts dieser wachsenden Euphorie scheint es jedoch erforderlich, diese Ergebnisse etwas differenzierter zu betrachten. Bis heute hat die Identifikation dieser neuen Risikofaktoren keine neuen Erkenntnisse zur Pathogenese der relevanten komplexen Krankheiten erbracht. Viele der assoziierten Marker wurden in nicht kodierenden Genomabschnitten oder in Genen mit unbekannter Funktion gefunden; in anderen Studien war es aufgrund der begrenzten Auflösung von Assoziationsstudien und Kopplungsanalysen nicht möglich, die verantwortlichen Sequenzvarianten genau zu kartieren.

Darüber hinaus führen die meisten dieser Faktoren nur zu einer geringen Veränderung des Wiederholungsrisikos, wobei eine zweifache Risikozunahme bereits die Ausnahme darstellt. Im Gegensatz dazu haben Geschwister eines Patienten mit komplexen Krankheiten wie Schizophrenie, Typ IDiabetes oder Lippen-Kiefer-Gaumenspalten ein gegenüber dem Populationsrisiko um den Faktor 10 bis 40 erhöhtes Wiederholungsrisiko. Die kürzlich identifizierten genetischen Risikofaktoren haben daher keinen diagnostischen und nur einen geringen prognostischen Wert. Dies wird sich erst dann ändern, wenn die meisten dieser genetischen Risikofaktoren identifiziert sind - und wenn die Typisierung aller dieser Faktoren Bestandteil der diagnostischen Routine geworden ist. Bis dahin ist es jedoch noch ein weiter Weg.

\section{Warum monogene Krankheiten systematisch aufgeklärt werden sollten.}

Neben der unzureichenden Markerdichte und zu kleinen Kohorten gibt es noch andere Probleme, welche die Suche nach genetischen Risikofaktoren für komplexe Krankheiten erschweren. Eines ist die genetische Heterogenität, welche für die Komplexität vieler multifaktorieller Störungen verantwortlich ist. Das extremste Beispiel dafür ist die geistige Behinderung (GB), die komplexe Krankheit mit den höchsten sozioökonomischen Kosten in den Staaten der ,Ersten Welt' (Roeleveld et al., 1997; Ropers und Hamel, 2005; Ropers, 2006). Etwa 300 bekannte Gendefekte gehen mit geistiger Behinderung einher, aber wahrscheinlich können Tausende verschiedener Gendefekte zur geistigen Behinderung führen. Verschiedene monogene Defekte hat man auch bei einer großen Vielzahl anderer komplexer Krankheiten gefunden, wie Alzheimer, Parkinson, Brust- und Dickdarmkrebs, Herzinfarkt und Bluthochdruck (Peltonen et al., 2006; Campion, 2001). Viele unserer heutigen Kenntnisse zur Pathogenese komplexer Krankheiten sind das Ergebnis monogener Unterformen. Es ist gut möglich, dass Neumutationen bei diesen Krankheiten sehr viel häufiger sind als bisher angenommen; aufgrund der kurzen evolutionären Lebensspanne dieser Mutationen kann man sie mit Hilfe von Assoziationsstudien nicht finden.

Die systematische Resequenzierung von Genen für monogene Krankheiten ist daher auch eine viel versprechende Strategie für die Identifikation von Risikofaktoren für komplexe Störungen. Allerdings sind monogene Krankheiten auch für sich genommen wichtig. Bis heute sind nur etwa 2000 der geschätzten 25000 Eiweiß kodierenden menschlichen Gene mit Krankheiten in Verbindung gebracht worden, und Krankheit verursachende Mutationen kennt man erst für weniger als 3400 Ein-Gen-Krankheiten (McKusick, 2007). Dabei handelt es sich jedoch sicher nur um die Spitze des Eisbergs. Viele Erbkrankheiten werden nicht als solche erkannt, insbesondere dann, wenn es sich um sporadische Fälle handelt. Schwere autosomal vererbte Krankheiten, die sich bereits in der Jugend manifestieren, sind jedoch meistens sporadisch, weil die betroffenen Patienten fast nie Kinder haben. Angesichts der in Deutschland sehr seltenen Blutsverwandtschaft von Ehepartnern und im Mittel nur 1,3 Kindern pro Familie werden auch die meisten rezessiven Krankheiten nur als isolierte Fälle in Erscheinung treten. Bei der Maus führen die meisten homozygoten Gendefekte zu phänotypischen Auffälligkeiten; nach vorliegenden Daten sind nur wenige Prozent dieser Tiere klinisch normal. Daher ist es sehr wahrscheinlich, dass die allermeisten menschlichen Gene, deren Defekte Krankheiten zur Folge haben, noch nicht entdeckt sind.

Bei der Maus werden die Auswirkungen von Defekten einzelner Genen auf den Phänotyp schon seit langem syste- 


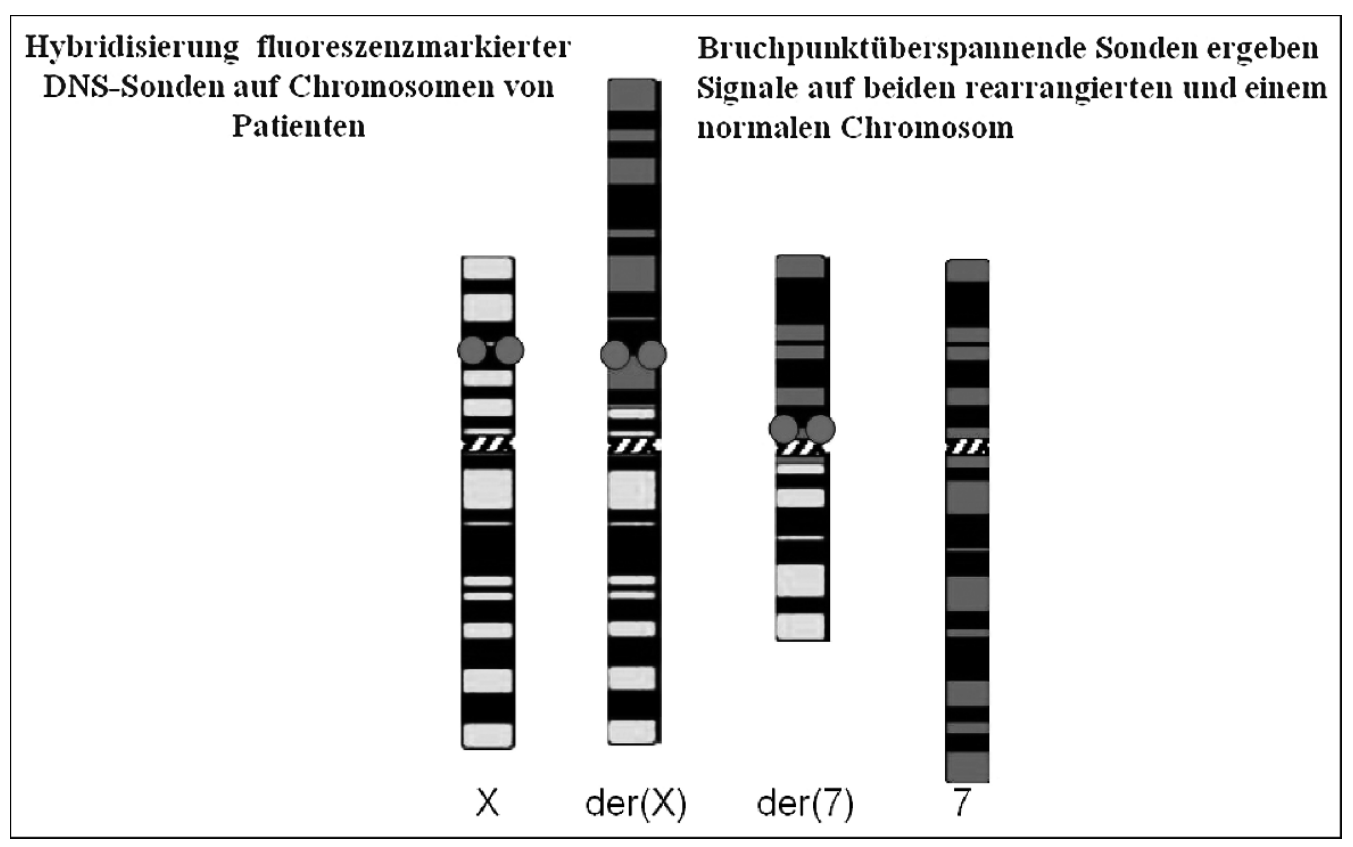

Abb. 1. Identifizierung von Krankheitsgenen durch Bruchpunktkartierung in Patienten mit balancierten Chromosomenveränderungen. matisch untersucht. Systematische Untersuchungen zur Aufklärung von monogenen Krankheiten beim Menschen sind hingegen bisher unterblieben. Dies ist besonders verwunderlich, weil für viele Krankheiten sogar die nahe verwandte Maus kein gutes Modell ist. Viele Untersuchungen haben gezeigt, dass orthologe Gendefekte in den beiden Spezies unterschiedliche phänotypische Folgen haben. Darüber hinaus ist es extrem schwierig, bestimmte komplexe Veränderungen, wie kognitive Störungen, im Mausmodell zu untersuchen.

All dies spricht dafür, die Anstrengungen zur Aufklärung von Ein-Gen-Krankheiten beim Menschen wesentlich zu verstärken (Antonarakis und Beckmann, 2006), zumal diese Untersuchungen durch die Verfügbarkeit der gesamten menschlichen Genomsequenz sehr viel einfacher geworden sind. Gegenwärtig gibt es eine ganze Reihe verschiedener Strategien zur Untersuchung von Mendelschen Krankheiten und mehrere dieser Strategien sind auch für systematische Untersuchungen an komplexen Krankheiten geeignet.

\section{Chromosomenaberrationen und Krankheiten}

Krankheitsassoziierte balancierte Chromosomenaberrationen (KBCA), welche Gene zertrennen oder auf andere Weise inaktivieren, stellen eine sichtbare Brücke zwischen menschlichen Phänotypen und Genotypen dar. Die systematische Bruchpunktkartierung und -charakterisierung in Patienten mit KBCA ist daher eine effiziente Strategie für die Aufklärung der molekularen Ursachen von genetisch bedingten Erkrankungen (Wirth et al., 1999; Bugge et al., 2000).

Neu aufgetretene KBCAs kann man durch konventionelle Karyotypisierung erkennen, und mit einer Häufigkeit von 1:2000 sind sie nicht selten. Etwa 6\% dieser Fälle sind mit klinischen Anomalien wie geistiger Behinderung (GB) mit oder ohne kongenitalen Anomalien assoziiert, die man in ungefähr der Hälfte dieser Fälle findet. Ein Vorteil dieses Ansatzes ist es, dass Bruchpunkte genau kartiert werden können, was das Auffinden der relevanten Gene erleichtert. In Zusammenarbeit mit einer dänischen Arbeitsgruppe ist es uns in den vergangenen zehn Jahren gelungen, eine große Zahl von Genen für GB und verwandte Krankheiten zu identifizeren, und seit einiger Zeit wird dieselbe Strategie auch von anderen Arbeitsgruppen verfolgt.

Die Comparative Genomische Hybridisierung mit Hilfe von DNA-Rastern, welche das menschliche Genom abdecken (Array-CGH), erlaubt die Suche nach submikroskopischen Deletionen und Duplikationen im gesamten menschlichen Genom. Mithilfe dieser Methode konnten in jüngster Zeit bei einer Vielzahl genetisch bedingter Störungen kleine Deletionen oder Duplikationen gefunden werden, und in mehreren Fällen gelang es sogar, die einzelnen Gene zu identifizieren, deren Deletion oder Duplikation für die betreffende Krankheit verantwortlich ist. Mikrodeletionen oder Duplikationen wurden bei etwa 7\% von Patienten mit nonsyndromaler GB identifiziert (de Vries et al., 2005), und 14\% einer unselektierten Kohorte von geistig behinderten Patienten mit normalem Karyotyp wiesen derartige Veränderungen auf (de Vries et al., 2007).

In jüngster Zeit konnte gezeigt werden, dass derartige submikroskopische Veränderungen nicht auf GB beschränkt sind, sondern auch bei Patienten mit Autismus, kongenitalen Herzfehlbildungen, Hirnfehlbildungen und anderen komplexen Krankheiten vorkommen. Die Identifikation solcher Veränderungen und ihre molekulare Charakterisierung bei Patienten mit monogenen und komplexen Krankheiten ist eine neue Dimension in der Identifikation von klinisch relevanten Gendefekten. 


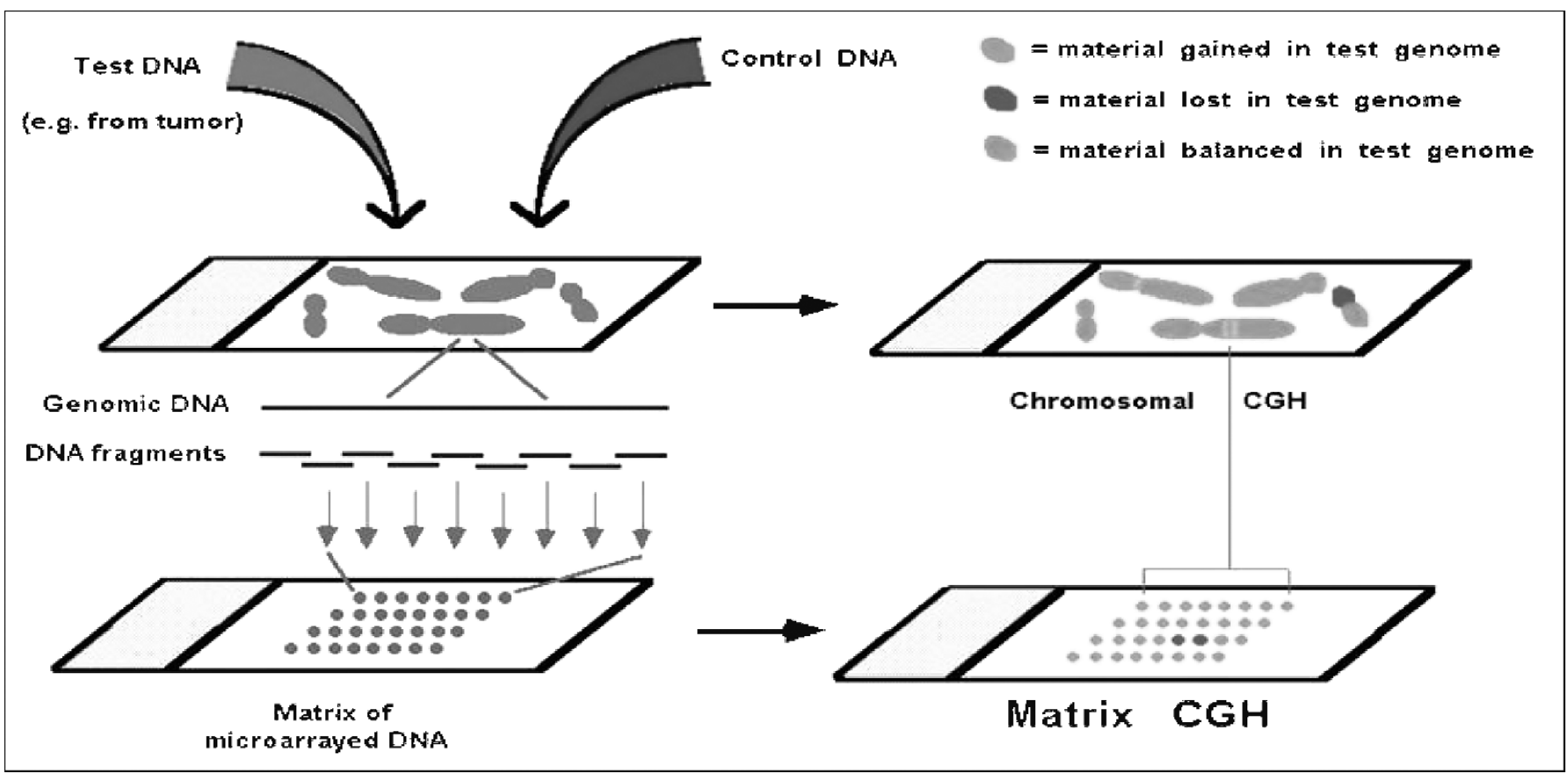

Abb. 2. Comparative Genomische Hybridisierung mit DNA-Chips erlaubt den Nachweis von submikroskopischen chromosomalen Veränderunge überall im Genom: neue Dimension für Diagnostik und Genomforschung.

\section{Mutationsscreening im großen Stil}

Die Suche nach Mutationen in Genen, die aufgrund ihrer Lage im Genom oder ihrer Funktion als Kandidaten für bestimmte Krankheiten in Frage kommen, ist eine andere naheliegende Strategie für die Identifikation von Krankheitsgenen. X-chromosomale Störungen sind plausible Ziele für derartige Strategien, weil man sie aufgrund ihres charakteristischen Erbgangs einfach erkennen kann. Auf diese Weise konnten viele der bekannten Ein-Gen-Krankheiten des Menschen dem XChromosom zugeordnet werden, und ungefähr 18\% der 900 bekannten X-chromosomalen Gene konnten bereits als Krankheitsgene identifiziert werden. Eine Option für die systematische Aufklärung der molekularen Grundlagen X-chromosomaler Krankheiten ist die Suche nach Mutationen in allen X-chromosomalen Genen. Ein illustratives Beispiel dafür ist das Projekt eines internationalen Konsortiums, alle im Hirn exprimierten X-chromosomalen Gene für Mutationen in einer großen Kohorte von Familien mit X-chromosomaler GB zu sequenzieren (Chiurazzi et al., 2008). Für andere Chromosomen ist dieser Ansatz jedoch weniger attraktiv, weil sie viel weniger dicht mit Krankheitsgenen bevölkert sind. Aufgrund der dafür erforderlichen enormen Sequenzierkapazität waren derartige Vorhaben bisher nur an wenigen großen Genomzentren möglich, jedoch wird sich dies dank der zunehmenden Verbreitung neuer Hochdurchsatz-Sequenziersysteme bald ändern.

\section{Autosomal rezessive Krankheiten verdienen mehr Aufmerksamkeit.}

Theoretische Überlegungen und empirische Daten sprechen dafür, dass die meisten der nicht aufgeklärten Erbkrankheiten einem autosomal-rezessiven Erbgang folgen, wie oben ausgeführt. Die Strategie der Wahl für die Identifizierung autosomal-rezessiver Gendefekte ist die Homozygotie-Kartierung in großen konsanguinen Familien (Najmabadi et al., 2007).

Solche Familien sind in industrialisierten Ländern jedoch außerordentlich selten, was die Tatsache erklärt, dass bis heute nur relativ wenige autosomal-rezessive Gendefekte bekannt sind. In arabischen und anderen, überwiegend islamischen Ländern sind $20 \%$ bis über $50 \%$ der Ehepartner jedoch blutsverwandt, und große Familien sind eher die Regel als die Ausnahme. In diesen Ländern ist die Häufigkeit von GB und kongenitalen Anomalien im Vergleich zu westlichen Ländern erhöht, was wenigstens zum Teil auf eine größere Häufigkeit rezessiver Störungen zurückgeführt wird (Bittles, 2001); in westlichen Ländern werden rezessive Krankheiten vermutlich häufig nicht als solche erkannt. In den meisten Ländern des sog. „Konsanguinitätsgürtels“, der von Marokko bis Pakistan reicht, sind systematische Untersuchungen zur Aufklärung der Ursachen rezessiver Krankheiten jedoch nicht oder nur mit Einschränkungen möglich. Aufgrund der komplementären Ressourcen spricht daher viel für eine internationale Kooperation zwischen diesen Ländern und westlichen Industrieländern. Ein Modell für derartige Interaktionen ist die vor fünf Jahren initiierte Zusammenarbeit unserer Arbeitsgruppe mit einer Gruppe im Iran, welche die Aufklärung rezessiver Formen der GB als Ziel hat. Bis heute konnten wir gemeinsam bereits 30 verschiedene Gene für autosomal-rezessive GB 


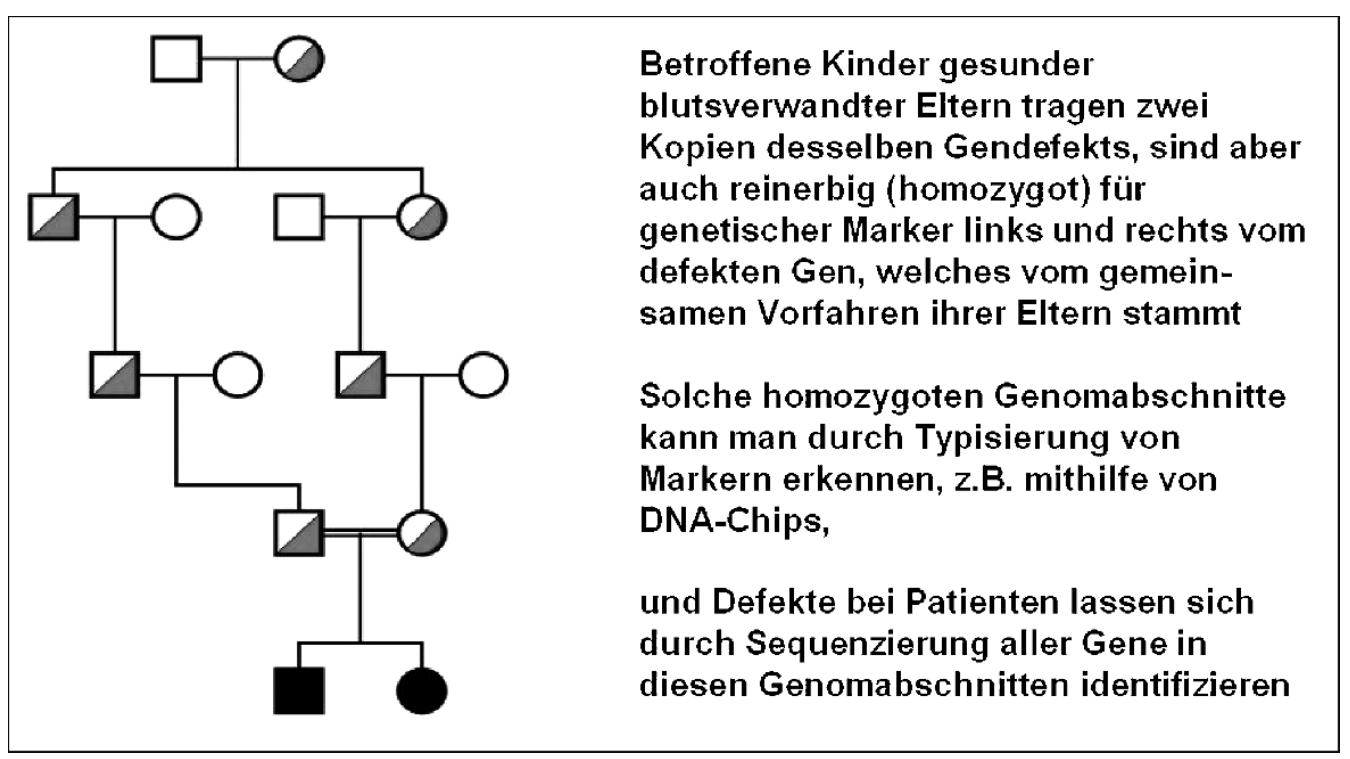

Abb. 3. Homozygotie-Kartierung in blutsverwandten Familien: Eine effiziente Strategie zur Aufklärung rezessiver Krankheiten. chromosomal lokalisieren und mehrere Gendefekte identifizieren. Bis zur Identifikation und molekularen Aufklärung aller autosomal-rezessiven Formen der GB, an der beim Menschen mehr als 1000 verschiedene Gene beteiligt sein können, ist es jedoch noch ein weiter Weg.

\section{Auswahl von Genkandidaten und Erkennung relevanter Mutationen}

Homozygote Genomabschnitte in konsanguinen Familien enthalten nicht selten Hunderte von Genen, weshalb die Suche nach den eigentlichen Mutationen außerordentlich langwierig und kostspielig sein kann, auch wenn es seit kurzem Software-Programme gibt, welche die Auswahl von Kandidatengenen anhand funktioneller Kriterien erleichtern (Aerts et al., 2006; Franke et al., 2006; Lage et al., 2007). Ein anderes Problem bei der Suche nach Krankheit verursachenden Mutationen ist die Tatsache, dass nicht alle diese Veränderungen einfach erkennbar sind. Obwohl große Datenbanken mit allen bisher bekannten Mutationen existieren, ist die klinische Relevanz neuer Missense-Mutationen noch immer recht schwierig, sogar wenn man Software-Programme zu Hilfe nimmt, welche diese Aufgabe erleichtern. Auch ist das Mutations-Screening heute meistens noch auf die Abschnitte von Genen beschränkt, welche für Proteine kodieren, weshalb Mutationen, die das Splicingmuster oder die Expression von Genen verändern, häufig übersehen werden. Mit der Einführung neuer, sehr viel kostengünstigerer Sequenziermethoden sollte sich dies jedoch bald ändern.

\section{Neue Sequenziertechnologien}

In den vergangenen 12 Monaten sind zwei Sequenziersysteme der neuen Generation zur Marktreife gelangt und ihre Einführung ist im Begriff, die Genomforschung zu revolutionie- ren (Bentley, 2006). Für diese neuen Verfahren zur Hochdurchsatz-Sequenzierung ist es zunächst erforderlich, die zu untersuchende DNS zu fragmentieren und die Fragmente klonal zu amplifizieren. Daran schließt sich bei dem von der Firma Roche vertriebenen System die parallele Pyrosequenzierung der amplifizierten Fragmente an, bei dem Gerät der Firma Illumina eine massiv parallele schrittweise Inkorporation fluoreszenzmarkierter Nukleotide. Daran schließt sich eine Visualisierung mit Hilfe empfindlicher Systeme zum Nachweis der betreffenden Licht- bzw. Fluoreszenzsignale an. Wie von Bentley (2006) ausführlich dargestellt, unterscheiden sich beide Systeme nicht nur im Hinblick auf die Länge der produzierten Einzelsequenzen, sondern auch in der Sequenzierkapazität und den Kosten der Sequenzierung. Mit Hilfe der kostengünstigeren Illumina-Technologie ist es jetzt möglich, den nicht repetitiven Anteil des gesamten menschlichen Genoms für wenig mehr als 100000 Dollar zu resequenzieren, über vier Größenordnungen weniger als die ungefähr drei Milliarden Dollar, welche bis zum Abschluss des Humangenomprojekts aufgewendet wurden. Bei linearer Fortschreibung dieses Trends ist zu erwarten, dass es in spätestens fünf Jahren möglich sein müßte, das gesamte menschliche Genom für 1000 Dollar zu sequenzieren, und tatsächlich könnte dies mit Hilfe neuer, in Entwicklung befindlicher Sequenziersysteme innerhalb dieses Zeitraumes Realität werden.

\section{Implikationen für die Aufklärung von genetisch bedingten Krankheiten}

Im Vergleich mit dem „Tausend-Dollar-Genom“ und seinen Implikationen für die Forschung und Krankenversorgung hat die zur Zeit stattfindende Verdrängung der konventionellen Sanger-Sequenzierung durch billigere und schnellere Resequenzierungstechniken bisher kaum öffentliches Aufsehen erregt. Für die Aufklärung von genetischen Krankheiten haben diese Entwicklungen jedoch weit reichende Konse- 
quenzen. Durch Einführung dieser Techniken wird die Sequenzierung indirekte (SNP-Typisierungs-)Methoden zur Identifizierung von Krankheitsgenen und genetischen Risikofaktoren zunehmend ersetzen. In Kombination mit neuen, effizienten Verfahren zur gezielten Anreicherung definierter Abschnitte des menschlichen Genoms (Albert und Oltvai, 2007) werden diese neuen Sequenziertechniken die Suche nach funktionell relevanten Mutationen in ausgedehnten physikalischen oder genetischen Genomintervallen ermöglichen. Zum ersten Mal wird es ökonomisch möglich werden, ganze Gene im großen Stil zu sequenzieren, einschließlich Promotorregionen, Introns und untranslatierten Abschnitten, in denen sich aller Wahrscheinlichkeit nach bisher unentdeckte pathogenetisch relevante Mutationen verbergen. Im Prinzip sollten diese neuen Verfahren zur Mutationserkennung viel zuverlässiger sein als die Resequenzierung mit Hilfe von DNA-Rastern, was eigene Untersuchungen bestätigen. Das bedeutet, dass die Einführung dieser neuartigen Hochdurchsatz-Sequenziertechniken auch ihren Einzug in die Diagnostik halten wird, sogar im Bereich der Zytogenetik. Zum Beispiel ist die Sequenzierung von sortierten Translokationschromosomen eine sehr schnelle und ökonomische Strategie zur Charakterisierung chromosomaler Bruchpunkte bei Patienten mit KBCAs (Chen et al., 2008). Eine Weiterentwicklung der o. g. Hochdurchsatz-Sequenziertechniken, die ,paired read'-Sequenzierung, erlaubt es inzwischen sogar, Mikrodeletionen, Duplikationen oder Inversionen durch Sequenzierung zu identifizieren, als vermutlich überlegene Alternative zur Array-CGH, welche die Zytogenetik erst vor kurzem revolutioniert hat.

\section{Schlußfolgerungen und Ausblick}

Die Einführung von DNA-Rastern zur hochauflösenden Typisierung von SNPs und neuartige Pooling-Strategien haben die Erkennung von wichtigen genetischen Risikofaktoren für komplexe Krankheiten sehr erleichtert, jedoch scheinen solche „Hauptgene“ sehr selten zu sein. Stattdessen wird es immer deutlicher, dass seltene Allele und evolutionär kurzlebige Mutationen eine wichtige Rolle in der Ätiologie komplexer Krankheiten spielen. Diese sind zudem viel heterogener als früher angenommen. Dies ist keine gute Nachricht für Pharmaunternehmen, die nach sog. „blockbuster drugs“ suchen, welche die meisten oder alle Patienten mit einer bestimmten häufigen Krankheit heilen können. Die gute Nachricht ist, dass die Unterteilung komplexer Krankheiten in viele verschiedene, häufig monogene Defekte die Chancen zur Aufklärung der zugrunde liegenden pathogenetischen Mechanismen deutlich verbessert hat.

Im Hinblick auf die verschiedenen effizienten Strategien zur Aufklärung von Gendefekten, einschließlich der Identifizierung von Mikrodeletionen und Duplikationen im ganzen Genom und die Einführung von Hochdurchsatz-Sequenzierverfahren läßt sich absehen, dass die Genomtypisierung nicht mehr lange der Flaschenhals dieser Untersuchungen sein wird. Angesichts der enormen genetischen Variabilität des menschlichen Genoms wird die Hauptaufgabe in Zukunft je- doch darin bestehen, die vergleichsweise wenigen Krankheit verursachenden Sequenzvarianten von den vielen funktionell neutralen zu unterscheiden. Dies wird die Untersuchung sehr großer Kohorten von Patienten und Kontrollen erfordern. Der Erfolg derartiger Studien wird jedoch entscheidend von der klinischen Charakterisierung der Patienten und von der Analyse und Interpretation der so ermittelten Daten abhängen. Neben der Phänotypisierung und der statistischen sowie bioinformatischen Analyse wird man dabei auch auf eine funktionelle Verifizierung der Untersuchungsergebnisse nicht verzichten können. Neben Krankheit verursachenden Mutationen und Risikofaktoren sollten diese Untersuchungen auch zur Identifikation von Sequenzvarianten führen, welche die Schwere und den Verlauf von genetisch bedingten Krankheiten beeinflussen. Schließlich werden diese Untersuchungen zur Aufklärung der für diese Krankheiten verantwortlichen pathogenetischen Mechanismen führen und damit neue therapeutische Möglichkeiten eröffnen, mit weit reichenden Konsequenzen für die Krankenversorgung allgemein.

\section{Literatur}

Aerts, S., Lambrechts, D., Maity, S., Van Loo, P., Coessens, B., De Smet, F., Tranchevent, L. C., De Moor, B., Marynen, P., Hassan, B., Carmeliet, P. und Moreau, Y. (2006) Gene prioritization through genomic data fusion. Nat Biotechnol 24:537-544.

Albert, R., und Oltvai, Z. N. (2007) Shaping specificity in signaling networks. Nat Genet 39:286-287.

Antonarakis, S. E. und Beckmann, J. S. (2006) Mendelian disorders deserve more attention. Nature Reviews Genetics 7:277-282.

Bentley, D. R. (2006) Whole-genome re-sequencing. Current Opinion in Genetics \& Development 16:545-552.

Bittles, A. H. (2001) Consanguinity and its relevance to clinical genetics. Clin Genet 60:89-98.

Bugge, M., Bruun-Petersen, G., Brøndum-Nielsen, K., Friedrich, U., Hansen, J., Jensen, G., Jensen, P. K. A., Kristoffersson, U., Lundsteen, C., Niebuhr, E., Rasmussen, K. R., Kirsten Rasmussen, K. und Tommerup, N. (2000) Disease associated balanced chromosome rearrangements: a resource for large scale genotype-phenotype delineation in man. J Med Genet 37:858-865.

Campion, D. (2001) Dissection génétique des maladies à hérédité complexe. médecine/sciences 17:1139-1148.

Chen, W., Kalscheuer, V., Tzschach, A., Menzel, C., Ullmann, R., Schulz, M., Erdogan, F., Li, N., Kijas, Z., Arkesteijn, G., Lopez Pajares, I., Goetz-Sothmann, M., Heinrich, U., Rost, I., Dufke, A., Grasshoff, U., Glaeser Glaeser, B., Vingron, M. und Ropers, H. H.(2008) Mapping translocation breakpoints by next-generation sequencing. Genome Research, published online 0: gr.076166.108v1-gr.076166.108.

Chiurazzi, P., Schwartz, C. E., Gecz, J. und Neri, G. (2008) XLMR genes: update 2007. Eur J Hum Genet 9:71-81.

Cohen, J. C., Kiss, R. S., Pertsemlidis, A., Marcel, Y. L., McPherson, R. und Hobbs, H. H. (2004) Multiple rare alleles contribute to low plasma levels of HDL cholesterol. Science 305:869-872.

de Vries, B. B. (2007) Presented at the 3rd International Meeting on Cryptic Chromosomal Rearrangements in Mental Retardation and Autism, Troina, Sicily, April 13, 2007.

de Vries, B. B., Pfundt, R., Leisink, M., Koolen, D. A., Vissers, L. E. L. M., Janssen, I. M., van Reijmersdal, S., Nillesen, W. M., Huys, E. H. L. P. G., de Leeuw, N., Smeets, D., Sistermans, E. A., Feuth, T., van Ravenswaaij-Arts, C. M. A., van Kessel, A. D., Schoenmakers, E. F. P. 
M., Brunner, H. G. und Veltman, J. A. (2005) Diagnostic genome profiling in mental retardation. Am J Hum Genet 77:606-616.

Dina, C., Meyre, D., Gallina, S., Durand, E., Körner, A., Jacobson, P., Carlsson, L. M. S., Kiess, W., Vatin, V., Lecoeur, C., Delplanque, J., Vaillant, E., Pattou, F., Ruiz, J., Weill, J., Levy-Marchal, C., Horber, F., Potoczna, N., Hercberg, S., Le Stunff, C., Bougnères, P., Kovacs, P., Marre, M., Balkau, B., Cauchi, S., Chèvre, J. C. und Froguel, P. (2007) Variation in FTO contributes to childhood obesity and severe adult obesity. Nat Genet 39:724-726.

Franke, L., van Bakel, H., Fokkens, L., de Jong, E. D., Egmont-Petersen, M. und Wijmenga, C. (2006) Reconstruction of a functional Human Gene Network, with an application for prioritizing positional candidate genes. Am J Hum Genet 78:1011-1025.

Frayling, T. M., Timpson, N. J., Weedon, M. N., Zeggini, E., Freathy, R. M., Lindgren, C. M., Perry, J. R. B., Elliott, K. S., Lango, H., Rayner, N. W., Shields, B., Harries, L. W., Barrett, J. C., Ellard, S., Groves, C. J., Knight, B., Patch, A.-M., Ness, A. R., Ebrahim, S., Lawlor, D. A., Ring, S. M., Ben-Shlomo, Y., Jarvelin, M.-R., Sovio, U., Bennett, A. J., Melzer, D., Ferrucci, L., Loos, R. J. F., Barroso, I., Wareham, N. J., Karpe, F., Owen, K. R., Cardon, L. R., Walker, M., Hitman, G. A., Palmer, C. N. A., Doney, A. S. F., Morris, A. D., Davey-Smith, G., The Wellcome Trust Case Control Consortium, Hattersley, A. T. und McCarthy, M. I. (2007) A common variant in the FTO gene is associated with body mass index and predisposes to childhood and adult obesity. Science 316:889-894.

Gudmundsson, J., Sulem, P., Manolescu, A., Amundadottir, L. T., Gudbjartsson, D., Helgason, A., Rafnar, T., Bergthorsson, J. T., Agnarsson, B. A., Baker, A., Sigurdsson, A., Benediktsdottir, K. R., Jakobsdottir, M., Xu, J., Blondal, T., Kostic, J., Sun, J., Ghosh, S., Stacey, S. N., Mouy, M., Saemundsdottir, J., Backman, V. M., Kristjansson, K., Tres, A., Partin, A. W., Albers-Akkers, M. T., Marcos, J. G.-I., Walsh, P. C., Swinkels, D. W., Navarrete, S., Isaacs, S. D., Aben, K. A., Graif, T., Cashy, J., Ruiz-Echarri, M., Wiley, K. E., Suarez, B. K., Witjes, J. A., Frigge, M., Ober, C., Jonsson, E., Einarsson, G. V., Mayordomo, J. I., Kiemeney, L. A., Isaacs, W. B., Catalona, W. J., Barkardottir, R. B., Gulcher, J. R., Thorsteinsdottir, U., Kong, A. und Stefansson, K. (2007) Genome-wide association study identifies a second prostate cancer susceptibility variant at $8 \mathrm{q} 24$. Nat Genet 39:631-63.

Guyer, M. S. und Collins, F. S. (1993) The Human Genome Project and the future of medicine. Am J Dis Child 147:1145-1152.

Haiman, C. A., Patterson, N., Freedman, M. L., Myers, S. R., Pike, M. C., Waliszewska, A., Neubauer, J., Tandon, A., Schirmer, C., McDonald, G. J., Greenway, S. C., Stram, D. O., le Marchand, L., Kolonel, L. N., Frasco, M., Wong, D., Pooler, L. C., Ardlie, K., Oakley-Girvan, I., Whittemore, A. S., Cooney, K. A., John, E. M., Ingles, S. A., Altshuler, D., Henderson, B. E. und Reich, D. (2007) Multiple regions within 8q24 independently affect risk for prostate cancer. Nat Genet 39:638-644.

Helgadottir, A., Thorleifsson, G., Manolescu, A., Gretarsdottir, S., Blondal, T., Jonasdottir, A., Jonasdottir, A., Sigurdsson, A., Baker, A., Palsson, A., Masson, G., Gudbjartsson, D. F., Magnusson, K. P., Andersen, K., Levey, A. I., Backman, V. M., Matthiasdottir, S., Jonsdottir, T., Palsson, S., Einarsdottir, H., Gunnarsdottir, S., Gylfason, A., Vaccarino, V., Hooper, W. C., Reilly, M. P., Granger, C. B., Austin, H., Rader, D. J., Shah, S. H., Quyyumi, A. A., Gulcher, J. R., Thorgeirsson, G., Thorsteinsdottir, U., Kong, A. und Stefansson, K. (2007) A common variant on chromosome 9p21 affects the risk of myocardial infarction. Science 316:1491-1493.

Kryukov, G. V., Pennacchio, L. A. und Sunyaev, S. R. (2007) Most rare missense alleles are deleterious in humans: implications for complex disease and association studies. Am J Hum Genet 80:727-739.

Lage, K., Karlberg, E. O., Størling, Z. M., Ólason, P. I., Pedersen, A. G., Rigina, O., Hinsby, A. M., Tuemer, Z., Pociot, F., Tommerup, N., Moreau, Y. und Brunak, S. (2007) A human phenome-interactome network of protein complexes implicated in genetic disorders. Nat Biotechnol 25:309-316.

McKusick, V. A. (2007) Mendelian inheritance in man and its online version, OMIM. Am J Hum Genet 80:588-604.

McPherson, R., Pertsemlidis, A., Kavaslar, N., Stewart, A., Roberts, R., Cox, D. R., Hinds, D. A., Pennacchio, L. A., Tybjaerg-Hansen, A., Folsom, A. R., Boerwinkle, E., Helen H. Hobbs; H. H. und Cohen, J. C. (2007) A common allele on chromosome 9 associated with coronary heart disease. Science 316:1488-1491.

Najmabadi, H., Motazacker, M. M., Garshasbi, M., Kahrizi, K., Tzschach, A., Chen, W., Behjati, F., Hadavi, V., Esmaeeli Nieh, S., Abedini, S. S., Vazifehmand, R., Firouzabadi, S. G., Payman, J., Falah, M., Seifati, S. M., Gruters A., Lenzner, S., Jensen, L. R., Rüschendorf, F., Kuss, A. W. und Ropers, H. H. (2007) Homozygosity mapping in consanguineous families reveals extreme heterogeneity of non-syndromic autosomal recessive mental retardation and identifies 8 novel gene loci. Hum Genet 121:43-48.

Pearson, J. V., Huentelman, M. J., Halperin, R. F., Tembe, W. D., Melquist, S., Homer, N., Brun, M., Szelinger, S., Coon, K. D., Zismann, V. L., Webster, J. A., Beach, T., Sando, S. B., Aasyl, J. O., Heun, R., Jessen, F., Kolsch, H., Tsolaki, M., Daniilidou, M., Reiman, E. M., Papassotiropoulos, A., Hutton, M., Stephan, D. A. und Craig, D. W. (2007) Identification of the genetic basis for complex disorders by use of pooling-based genomewide single-nucleotide-polymorphism association studies. Am J Hum Genet 80:126-139.

Peltonen, L., Perola, M., Naukkarinen, J. und Palotie, A. (2006) Lessons from studying monogenic disease for common disease. Hum Mol Genet 15:R67-R74.

Rioux, J. D., Xavier, R. J., Taylor, K. D., Silverberg, M. S., Goyette, P., Huett, A., Green, T., Kuballa, P., Barmada, M. M., Datta, L. W., Shugart, Y. Y., Griffiths, A. M., Targan, S. R., Ippoliti, A. F., Bernard, E. J., Mei, L., Nicolae, D. L., Regueiro, M., Schumm, L. P., Steinhart, A. H., Rotter, J. I., Duerr, R. H., Cho, J. H., Daly, M. J. und Brant, S. R. (2007) Genome-wide association study identifies new susceptibility loci for Crohn disease and implicates autophagy in disease pathogenesis. Nat Genet 39:596-604.

Roeleveld, N., Zielhuis, G. A. und Gabreels, F. (1997) The prevalence of mental retardation: a critical review of recent literature. Dev Med Child Neurol 39:125-132.

Ropers, H. H. (2006) X-linked mental retardation: many genes for a complex disorder. Current Opinion in Genetics \& Development 16:1-10.

Ropers, H. H. (2007) New perspectives for the elucidation of genetic disorders. Am J Hum Genet 81:199-207.

Ropers, H. H. und Hamel, B. C. (2005) X-linked mental retardation. Nat Rev Genet 6:46-57.

Wirth, J., Nothwang, H. G., van der Maarel, S., Menzel, C., Borck, G., Lopez-Pajares, I., Brøndum-Nielsen, K., Tommerup, N., Bugge, M., Ropers, H. H. und Haaf, T. (1999) Systematic characterisation of disease associated balanced chromosome rearrangements by FISH: cytogenetically and genetically anchored YACs identify microdeletions and candidate regions for mental retardation genes. J Med Genet 36:271-278.

Yeager, M., Orr, N., Hayes, R. B., Jacobs, K. B., Kraft, P., Wacholder, S., Minichiello, M. J., Fearnhead, P., Yu, K., Chatterjee, N., Wang, Z., Welch, R., Staats, B. J., Calle, E. E., Feigelson, H. S., Thun, M. J., Rodriguez, C., Albanes, D., Virtamo, J., Weinstein, S., Schumacher, F. R., Giovannucci, E., Willett, W. C., Cancel-Tassin, G., Cussenot, O., Valeri, A., Andriole, G. L., Gelmann, E. P., Tucker, M., Gerhard, D. S., Fraumeni, J. F., Hoover, R., Hunter, D. J., Chanock, S. J. und Thomas, G. (2007) Genome-wide association study of prostate cancer identifies a second risk locus at 8q24. Nat Genet 39:645-649.

Zaitlen, N., Kang, H. M., Eskin, E. und Halperin, E. (2007) Leveraging the HapMap correlation structure in association studies. Am J Hum Genet 80:683-691. 